European Journal of Mechanics - B/Fluids

November 2018, Volume 72 Pages 471-484

http://dx.doi.org/10.1016/i.euromechflu.2018.07.014

http://archimer.ifremer.fr/doc/00453/56423/

(c) 2018 Elsevier Masson SAS. All rights reserved.

\title{
A 3D study of the bubble sweep-down phenomenon around a $1 / 30$ scale ship model
}

\author{
Mallat Bachar ${ }^{1}$, Germain Grégory ${ }^{1,{ }^{*}}$, Billard Jean-Yves ${ }^{2}$, Gaurier Benoit ${ }^{1}$ \\ ${ }^{1}$ IFREMER, Marine Structure Laboratory, 150 Quai Gambetta 62200 Boulogne sur Mer, France \\ 2 IRENAV, Ecole Navale, CC 600 - Lanveoc, 29240 Brest Cedex 9, France \\ * Corresponding author : Grégory Germain, email address : ggermain@ifremer.fr
}

\begin{abstract}
:
The bubble sweep-down phenomenon around oceanographic research vessels generates acoustic disturbances. A specific experimental protocol in a wave and current circulating tank is used to study this phenomenon around a 1/30 scale ship model in a configuration with current, waves and ship motions. 3D visualizations of the bubble clouds and Stereo-PIV (Particle Image Velocimetry) measurements results, both obtained in the bow vicinity of the ship model, are presented in this paper. The behaviour of the breaking wave with bubble generation is studied and the spatial evolution of the bubble clouds is investigated. It is demonstrated that the bubble cloud generation is mainly conditioned by the ascending vertical velocity of the flow near the bow. It is observed that the bubble clouds are propagated in the flow direction with a horizontal expansion which is non negligeable, reaching a close position to the hull. The overall results provide new elements for the study and the understanding of the phenomenon compared with previous 2D studies.
\end{abstract}

\section{Highlights}

Air entrainment is studied around a 1/30 Scale Ship Model. $>$ 3D bubble cloud characteristics are determined. PIV measurements and POD analysis allow to study the air bubble generation.

Keywords : Bubble sweep-down, Breaking wave, Bow wave, Fluid-structure interaction, Flume tank 


\section{Introduction}

The bubble sweep-down phenomenon is a widely well known phenomenon that has been experienced to some degree on nearly every research vessel.

${ }^{1}$ Corresponding author. E-mail: ggermain@ifremer.fr 
It has detrimental effects on scientific sonar systems. On many specialized vessels, such as oceanographic survey and research vessels, warships and fisheries survey boats fitted with underwater acoustic transducers, bubble-sweep down can significantly degrade the transducer performance. The causes are less widely understood. This phenomenon is separated into two consequent events : the first one is the formation of bubbles by breaking waves coming from natural wind or the bow entry into the water, and the second one is the training of these bubbles by a path backwards along the ship hull and under the transducers which disrupt the acoustic signals and may result in a considerable reduction of the ships productivity.

This phenomenon has affected the acoustic surveys for many years [Dalen et al. (1981)] and is still a significant issue [Delacroix et al. (2016), Shabangu et al. (2014)]. The reason is that neither towing tank trials nor numerical simulations [Moraga et al. (2008)] are able to properly address this phenomenon [Johansen (2010)]. The experimental characterization of bubble generation by the breaking bow waves of a ship are scarse. The behaviour of these waves, depending on the bow geometry and the Froude number, have been studied by Noblesse et al. [Noblesse et al. (2008), Noblesse et al. (2013)] and Delhommeau et al. (2009). However these studies focused on bow wave in calm water for thin and fast ships. The influence of sea states and motions have not been considered, even if some attempts have been made by [Castro et al. (2016)]. Thus, this non-trivial phenomenon requires the development of a specific methodology to study it better.

While some differences can occure on air bubble generation and propagation between model and real scales due to Webber effects, flow measurements are representative at real scale thanks to the Froude similitude. For many years, Pitot tubes have been used to estimate the local flow velocity from pressure measurements. Kim et al. (2001) measured the forward resistance, waves and flow velocity (through Pitot tubes) around different models with bulb in a towing tank. Recently, numerous studies have focused on the characterization of the flow around ship hulls using Particle Image Velocimetry measurements in towing tanks [Chen et al. (2006)], such as in circulating tanks [Lee et al. (2003), Lee et al; (2009)] and cavitation tunnels [Paik et al. (2007)]. In particular, the area near the bow has been investigated by optical methods in order to characterize the deformation of the free surface [Gomit et al. (2013), Gomit et al. (2015)]. This deformation is a significant parameter in our study because it acts strongly on the bubble generation. The optical method of stereo-refraction developed in [Gomit et al. (2013)] 
was tested around the classic bow of the French oceanographic research vessel Pourquoi pas? (Pp?) in our wave and current circulating tank. This method is based on the apparent displacement of a known random pattern particles illuminated by a laser sheet located underneath the surface. This pattern serves as a reference observed by two different points of view. Unfortunately, the interaction between the wave and the current is at the origin of the appearance of wrinkles and capillary waves at the free surface which make it impossible to detect the PIV particles by the cameras positioned above the free surface. The free surface can not be characterized by this method.

Since the diphasic problem is extremely difficult to handle properly with the common tools, a specific experimental protocol has been developed in a circulating tank, allowing the visualization of the bubble sweep-down phenomenon around a specific ship bow [Delacroix et al. (Part 1 - 2016)]. This protocol is based on the simulation of conditions under which bubble sweepdown can be observed at full scale. In [Delacroix et al. (Part 1 - 2016)], the phenomenon has been investigated in two dimensions on a plane illuminated by a vertical laser sheet near the bow of the ship. Occurrence frequency of bubble clouds, bubble clouds dynamic in terms of area, depth and vertical velocity and PIV data analysis have been performed in this plane allowing the characterization of the phenomenon. However, this study is still limited because of the complexity of the phenomenon occuring in three dimensions. Thus, the development of an experimental set-up allowing to identify where these bubbles are really located in the depth of field (in the horizontal plane) is a substantial issue in order to obtain a 3D knowledge of the bubble sweepdown phenomenon.

The objective of this work is to present the experimental protocol that we recently conducted for a 3D characterization of the bubble sweep-down phenomenon around a 1/30 scale ship model. The first part of this paper presents the experimental set-up allowing the reproduction of the phenomenon in a flume tank. The video system used to characterize the flow is then described. The second part of this paper is devoted to the characterization of bubble clouds around the ship model. The evolution of the bow wave angle is investigated when the phenomenon occurs. The 3D reconstruction and evolution of three types of bubble clouds are presented and analysed. The final part of this paper focuses on the Stereo-PIV measurements followed by Proper Orthogonal Decomposition (POD) analysis in the bow vicinity to highlight the behaviour of the breaking wave during wave periods with and without 
bubbles.

\section{Experimental set-up}

\subsection{Facilities and ship model}

Experiments have been carried out at the Ifremer (French Research Institute for Exploration and Exploitation of the Sea) wave and current flume tank. The tank working section is $18 \mathrm{~m}$ long by $4 \mathrm{~m}$ wide and $2 \mathrm{~m}$ deep. The streamwise flow velocity range is $\mathrm{U}=0.1$ to $2.2 \mathrm{~m} / \mathrm{s}$. A wave generator (figure 1) generates regular and irregular waves with a frequency range between $\mathrm{f}=0.5$ and $2 \mathrm{~Hz}$ and a maximum height of $300 \mathrm{~mm}$ with a current speed up to $0.8 \mathrm{~m} / \mathrm{s}$. It can be easily moved between an upstream or a downstream surface position to create waves propagating with or against the current. Measurements revealed that the resulting reflection coefficient is lower than $12 \%$ for all the usual frequencies and amplitudes. A side observation window of $8 * 2 \mathrm{~m}^{2}$ placed on one side of the tank allows users to observe the behavior of the ship model during trials. In such facility, the model has been fixed on a motion generator system (hexapod) able to generate all kinds of motions. The free surface elevation has been measured by wave gauges placed upstream of the ship bow.

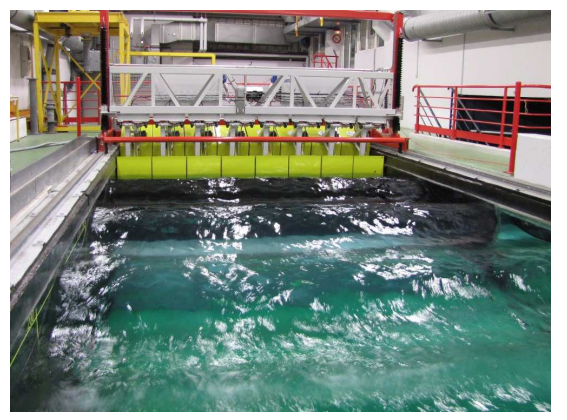

Figure 1: View of the wave generator system with regular waves and current.

To reproduce the bubble sweep-down phenomenon observed on the $P p$ ? during the acoustic survey Essbulles performed in 2005, a 1/30 scale ship model has been considered. The main conditions during this survey are: a ship speed of 8 knots, a wavelength of $109 \mathrm{~m}$, a significant wave height Hs of $2.8 \mathrm{~m}$, and a wave period $T_{p}$ of $8.4 \mathrm{~s}$. To reproduce navigation conditions and sea state encountered during the sea survey, the Froude similitude $\left(F r^{2}=\right.$ 
$V^{2} / g . L p p$ where $\mathrm{V}$ is the ship speed, $\mathrm{g}$ is the gravity and Lpp is model length) must be respected. Furthermore, the characteristics of the regular waves in the flume tank is calculated to conserve the energy density of irregular waves at sea. The energy density of irregular waves is given by the following expression:

$$
E=\rho g m_{0}\left[J / m^{2}\right], \text { with } H_{s}=4 \sqrt{m_{0}}
$$

where $H_{s}$ is the mean wave height (trough to crest) of the highest third of the waves $\left(H_{1 / 3}\right)$ and $m_{0}$ is the zero order moment of the wave energy spectra. For sinusoidal waves:

$$
E=\frac{1}{2} \rho g A^{2}, \text { where A is the amplitude }(A=H / 2) .
$$

To observe energy density conservation, one must write:

$$
\begin{gathered}
m_{0}=\frac{1}{2} A^{2} \\
\text { so } A=\frac{\sqrt{2} H_{s}}{4} \text { and } H=\frac{\sqrt{2} H_{s}}{2}
\end{gathered}
$$

For $H_{s}=2.8 m$ the sinusoidal waves with the same energy density will have a wave height $H=2.0 \mathrm{~m}$. The period $T$ will be taken equal to $T_{p}$. Therefore, the flow velocity in the flume tank is fixed at $0.75 \mathrm{~m} / \mathrm{s}$ and the wave height at $33 \mathrm{~mm}$ with a frequency of $0.85 \mathrm{~Hz}$. The characteristics of the the $P p$ ? at model and full scales are resumed in the table 1. Figure 2 presents the model with a side and front views. The model is fixed on a motion generator (hexapod) enabling the reproduction of the ship motion submitted to wave effects. Trials have been conducted on the front part only ( $1 / 3$ of the model) to avoid perturbations coming from the model stern. The experimental setup is summarized in figure 3 .

\subsection{Instrumentation and Method}

Two kinds of measurements have been carried out around the bow of the ship model where the bubble sweep-down phenomenon takes place. The first one is a 3D visualization of the phenomenon by video processing for the characterization of the bubble clouds and the bow wave dynamic. The second one is Stereo-PIV measurements for flow analysis. For both kind of measurements, Hi-sense CCD cameras of 1600x1200 pixels resolution and 

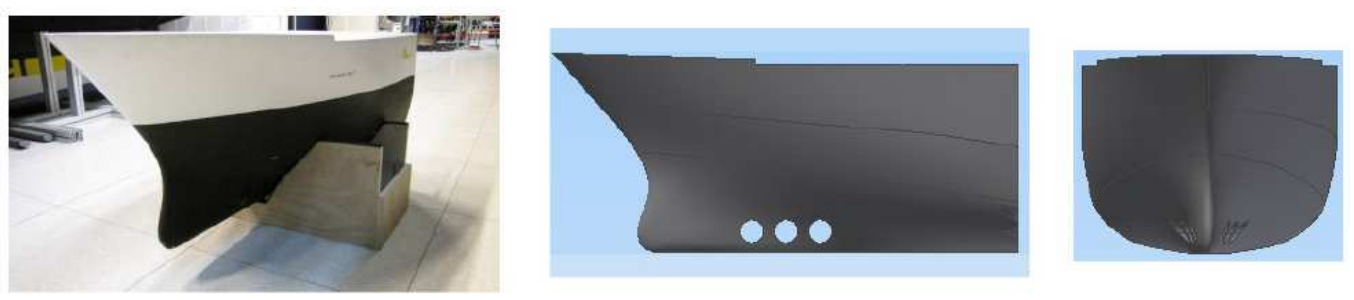

Figure 2: View of the $1 / 30$ scale ship model of $P p$ ?

\begin{tabular}{|c|c|c|}
\hline & \multicolumn{2}{|c|}{ Pourquoi pas? } \\
\hline & model scale & full scale \\
\hline $\operatorname{Lpp}(m)$ & 3.13 & 94 \\
\hline Beam $(\mathrm{m})$ & 0.67 & 20 \\
\hline Draft $(\mathrm{m})$ & 0.182 & 5.46 \\
\hline$C_{B}$ & 0.577 & 0.577 \\
\hline
\end{tabular}

Table 1: The characteristics of the $P p$ ? at model and full scales.
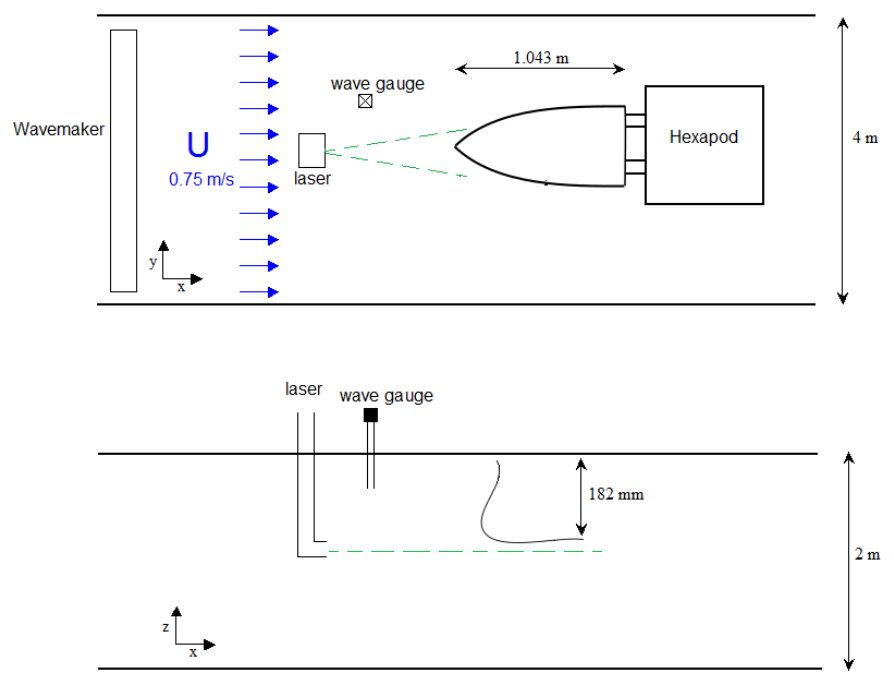

Figure 3: Schematic view of the experimental set-up in the wave and current circulating tank, top and side view.

different focal lenses have been used. Illumination has been provided by a standard, frequency-doubled, double-cavity Nd:YAG laser with a pulse 
energy of $200 \mathrm{~mJ}$ per pulse using an excitation wavelength of $532 \mathrm{~nm}$ instead of a $120 \mathrm{~mJ}$ laser for previous 2D studies. Silver-coated glass particles of $10 \mu \mathrm{m}$ diameter have been used to seed the tank.

\subsubsection{The 3D visualization of the phenomenon}

Three cameras have been disposed around the ship model (figure 4) in order to characterize and to quantify the propagation of air bubbles around the ship bow from different points of view. The first one (cam2 with a focal lens of $25 \mathrm{~mm}$ ) is installed above the free surface in order to visualize the breaking wave phenomenon with a physical dimension plane of $512 * 384 \mathrm{~mm}^{2}$. The second one (cam3 with a focal lens of $60 \mathrm{~mm}$ ) is located behind the side flume tank observation window allowing the visualization of air bubbles propagating along the ship hull with a physical dimension plane of $635 *$ $476 \mathrm{~mm}^{2}$. The third one (cam1 with a focal lens of $20 \mathrm{~mm}$ ) is located at the bottom of the tank in order to analyse the transverse expansion of the bubble clouds. The distance between the cam1 and the horizontal laser sheet is $1.6 \mathrm{~m}$; the cam 1 is located perpendicularly to the laser sheet. The physical dimension of this measured plane is $621.1 * 461.1 \mathrm{~mm}^{2}$. This camera has two objectives: i) the identification of the location of the bubbles in the depth of field in order to reconstruct the bubble cloud in 3 dimensions as we will see in the following parts of this paper; ii) the investigation of the behaviour of the bow wave. All cameras have been synchronized with the same system. Figure 4 presents the locations of instrumentations used in this study: the three cameras, the horizontal laser sheet and the wave gauges. The pulse energy of $200 \mathrm{~mJ}$ of the horizontal laser sheet has allowed a sufficient contrast

for the cam 1 at the bottom of the tank, unobtainable with a pulse energy of $120 \mathrm{~mJ}$ in previous study [Delacroix et al. (Part 1 - 2016)].

As mentioned above, previous studies have been done to characterize the bubble sweep-down phenomenon in 2 dimensions [Delacroix et al. (Part 1 2016)]. Illumination has been provided vertically on a plane in the vicinity of the ship bow (see figure 5) and images have been taken only by one camera behind the observation window allowing the visualization and propagation of air bubbles along the ship hull. Figure 6 presents an example of an image taken in such experiments where a bubble cloud is generated. Occurrence frequency of bubble clouds and bubble cloud dynamics have been investigated. Bubble clouds have been detected on the raw images and analysed by a gray scale level in order to calculate in $2 \mathrm{D}$ the area, depth and velocity of bubble clouds. However, all these results [Delacroix et al. (Part 1 - 2016), 


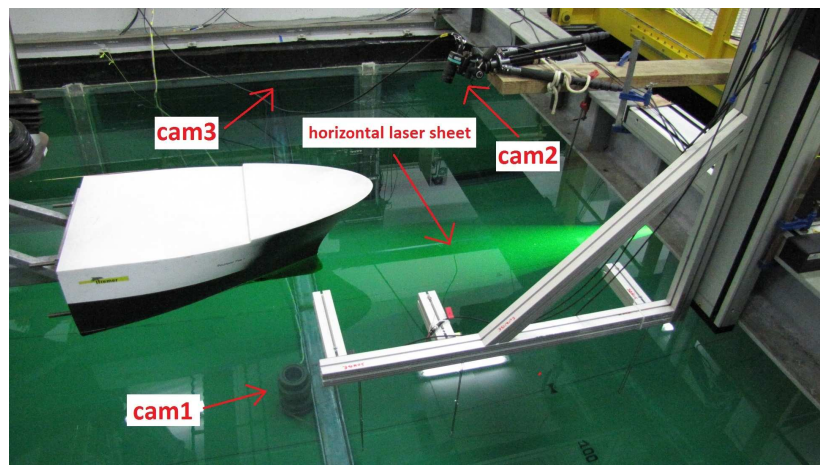

Figure 4: View of the experimental set-up: locations of the three cameras, the horizontal laser sheet and the wave gauges.

Delacroix et al. (Part 2 - 2016)] are performed without knowing the bubble positions in the depth of field (see figure 6).

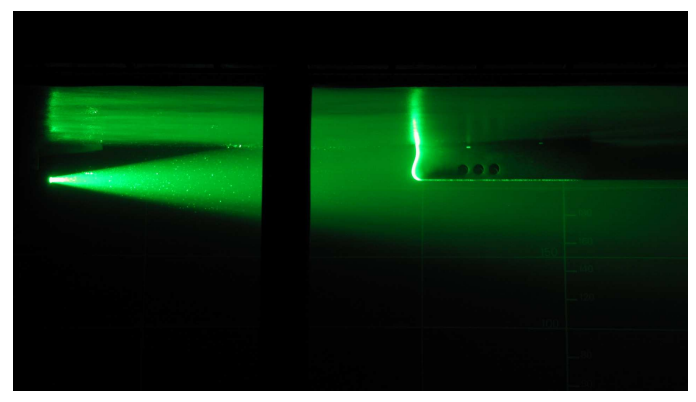

Figure 5: The vertical laser sheet around the classic bow of $P p$ ?

A precise characterization of the bubble sweep-down phenomenon imposes to know exactly where are the bubbles in the volume of measure. For that purpose, three cameras are used as mentioned in the experimental set-up description to visualize the bubble clouds from different points of view with a horizontal illumination plane. Figure 7 shows an example of a bubble cloud taken by the cam 1 and cam 3 around the classic bow of the $P p$ ? From the camera fixed at the bottom of the tank (cam1), we visualize the position of bubbles transversely in width. It is the main information that we had not in previous 2 dimensions study. With this information, we are now able to identify where bubbles shown from the side camera (cam3) are located breadthwise. In order to reconstruct the evolution of the bubble cloud in 3D, we proceed as follows: 


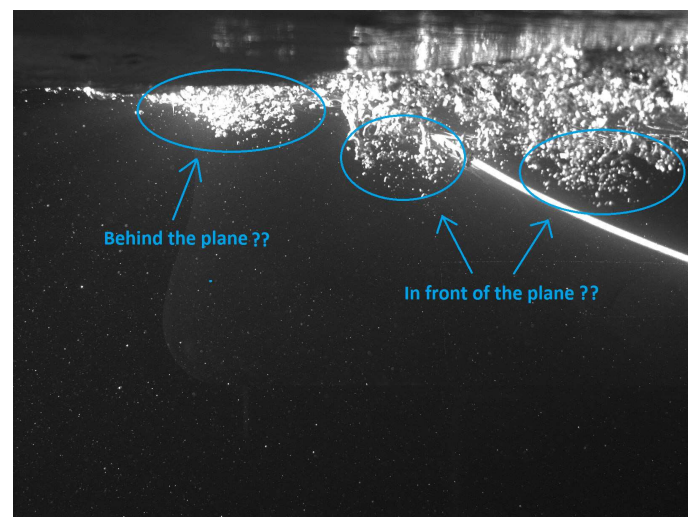

Figure 6: A raw image with bubble cloud around the classic bow of $P p$ ? in the 2 dimensions study. The illuminated plane trace is also visible on the bow.

- The raw images of cam1 and cam3 are converted to binary images taking into account a first gray level (see figure 7 ). The binary images enable to detect the free surface and the bow model location. A first region of the bubble cloud is then detected. A second loop with a second gray level threshold is applied in order to maximize the bubbles detection in the restricted region. Different thresholds have been tested in order to detect the bubble cloud accurately and it has been shown that the threshold variations have very little influence on the results presented in the following [Delacroix et al. (Part 2 - 2016)];

- For several locations along the model in the current direction $X_{i}$, we detect the minima and the maxima of the white area, for instance: $Z_{\text {mini }}, Z_{\text {maxi }}, Y_{\text {mini }}, Y_{\text {maxi }}$ (see figure 7 );

- From this detection, we construct an ellipse for each $\mathrm{X}$ position. The coordinate of the ellipse center is $\left(X_{i},\left(\left(Y_{\min }\right)_{i}+\left(Y_{\max }\right)_{i}\right) / 2,\left(\left(Z_{\text {min }}\right)_{i}+\right.\right.$ $\left.\left.\left(Z_{\max }\right)_{i}\right) / 2\right)$. The length of the semi-major axis and semi-minor axis is $\left(\left(Y_{\min }\right)_{i}+\left(Y_{\max }\right)_{i}\right) / 2$ and $\left(\left(Z_{\min }\right)_{i}+\left(Z_{\max }\right)_{i}\right) / 2$ respectively (see figure 8);

- The bubble cloud volume is drawing with ellipse connections. The choice of the ellipse shape permit to encompass the majority of bubbles without over estimation of the bubble area. 

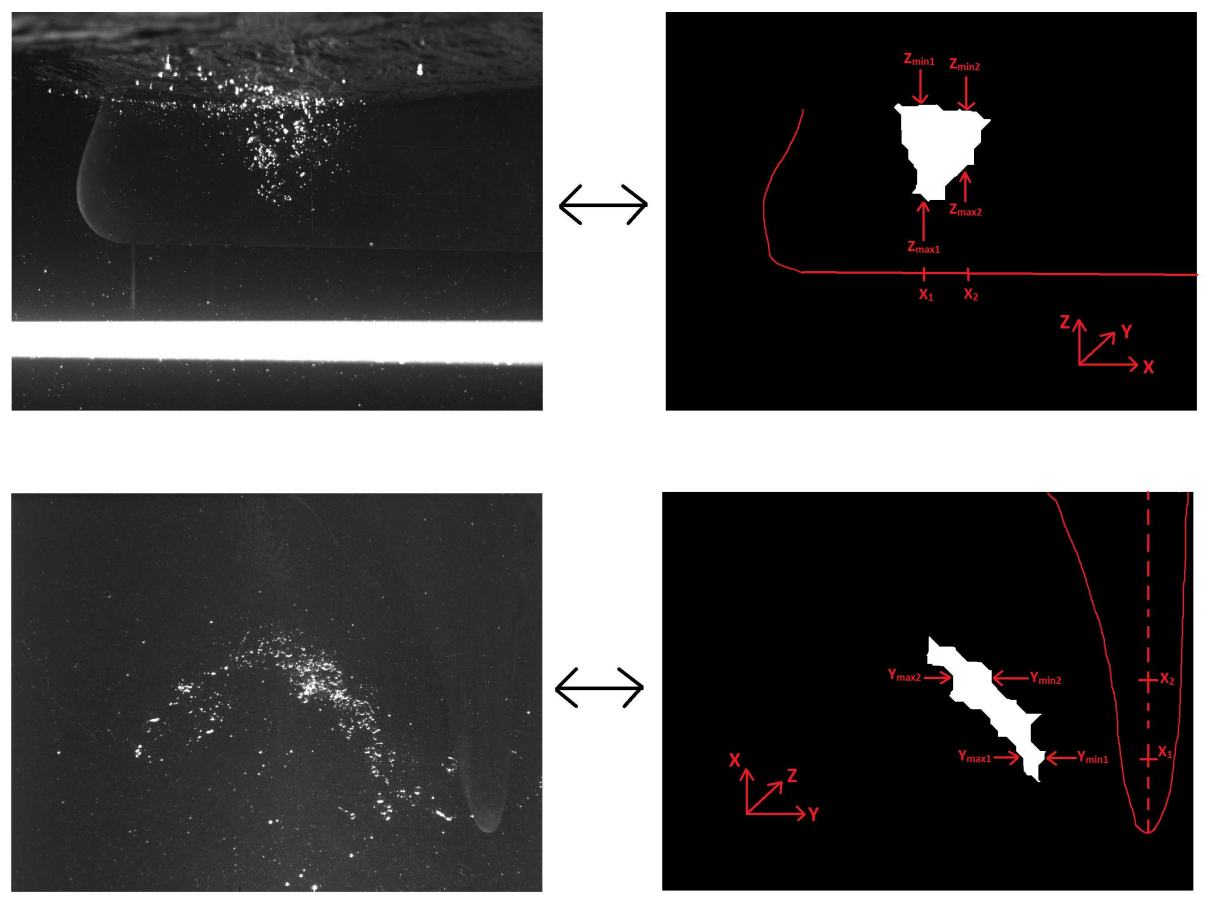

Figure 7: A selected bubble cloud and its detecion shown from cam 3 (top) and cam1 (bottom).

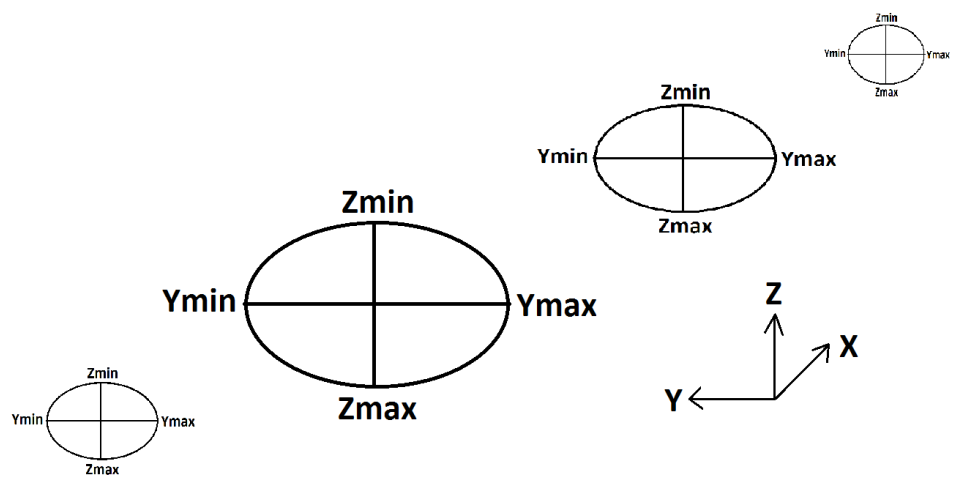

Figure 8: The decomposition of a bubble cloud into ellipses to reconstruct it in 3D.

\subsubsection{Stereo-PIV measurements}

Stereo-PIV measurements have also been carried out around the classic bow of the $P p$ ? to study the link between the hydrodynamic flow field 
and bubble generation. With this system, the three velocity components are investigated. The principle of the stereo-PIV is based on the ability to evaluate, with the least possible error created by distortions, the displacement in depth of the particles in the laser sheet with a thickness of about $6 \mathrm{~mm}$. It is thanks to this thickness that we can evaluate the transverse component of the velocity $\mathrm{V}$ directed towards the observation window.

Figure 10 presents the Stereo-PIV system composed of a vertical laser sheet and two Hi-sense CCD cameras, with a focal lense of $60 \mathrm{~mm}$. The time between pulses is fixed to $\Delta t=800 \mu \mathrm{s}$. The distance between the two cameras is $1440 \mathrm{~mm}$ and the distance between the cameras and the vertical laser sheet is $2.5 \mathrm{~m}$. The angle between the 2 cameras is $33 \mathrm{deg}$ as shown in figure 9. Measurements have been yielded during 5 minutes. The StereoPIV parameters are presented in the Table 2. Instantaneous velocity vector fields have been obtained using a Cross correlation PIV algorithm with an interrogation window size of 32x32 pixels followed by a Universal Outlier Detection (UOD).

The accuracy of the evaluation of the transverse velocity component is linked to the calibration required by the installation of a Scheimpflug support used during these trials. The calibration has been done with a $2 \mathrm{D}$ pattern (see figure 9 ) positionned at different locations in the vertical plane at $\mathrm{y}=$ $\{-4 ;-2 ; 0 ; 2 ; 4\}$.
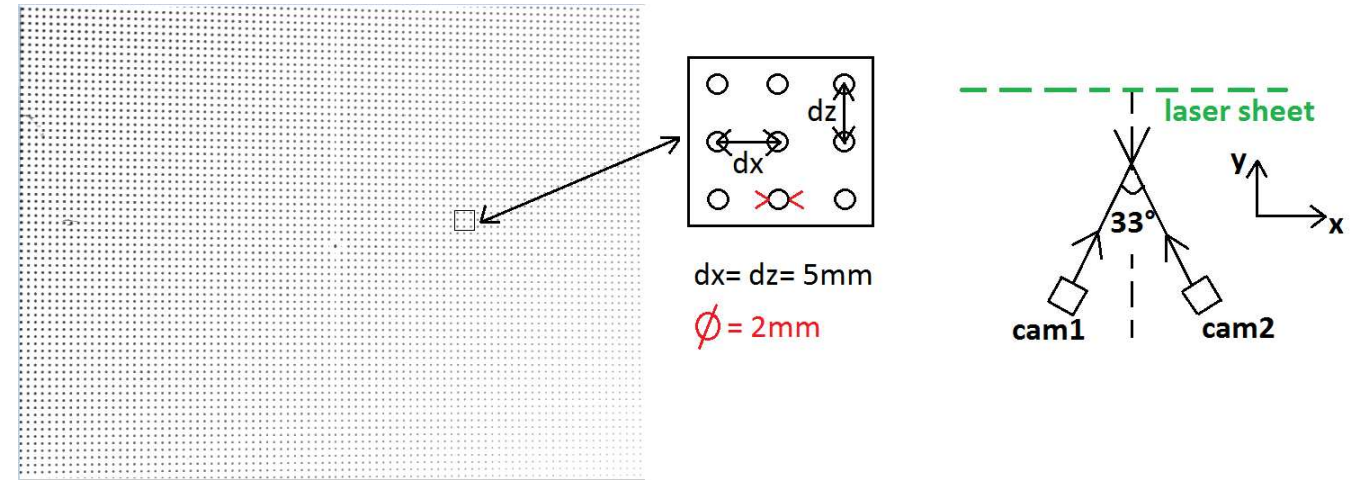

Figure 9: The 2D pattern for calibration (left). The orientation of the two cameras (right).

Stereo PIV measurements have been performed in the symmetrical plane $\mathrm{y}=0 \mathrm{~mm}$ in the front of the bow in order to investigate the flow in the area where breaking waves take place. In this zone, few bubbles are observed but masked by the automatic dynamic mask of the free surface [Dussol et al. 


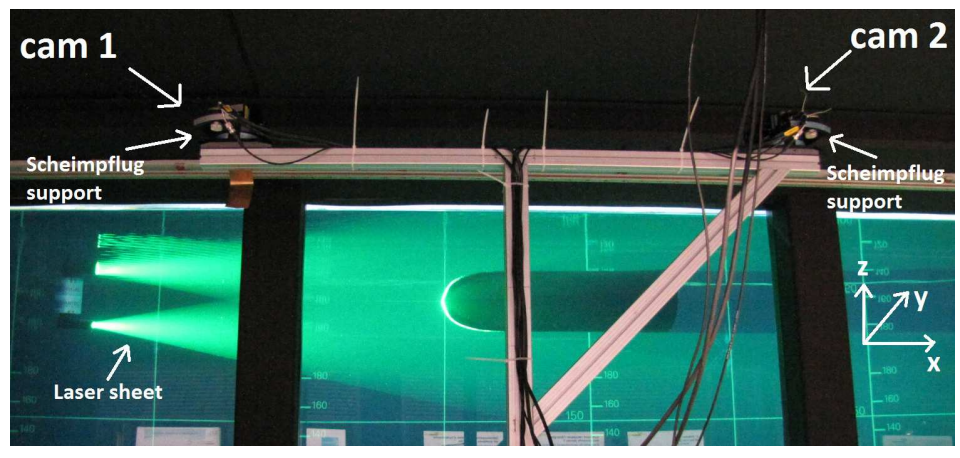

Figure 10: Presentation of the Stereo-PIV system.

\begin{tabular}{|c|c|}
\hline Parameters & Database \\
\hline Physical dimension (Stereo-PIV plane) & $500.7 \times 358.4 \mathrm{~mm}^{2}$ \\
Number of grid points & $\left(n_{x}, n_{z}\right)=(99,74)$ \\
Spatial discretization & $d x=d z=5 \mathrm{~mm}$ \\
Pixel discretization & $d p x=d p z=0.31 \mathrm{~mm}$ \\
Pixel and spatial discretization & $d x \approx 16 d p x$ \\
Average reprojection error of calibration & $1.8 * 10^{-1}$ pixels \\
\hline Sampling frequency $f_{e}(\mathrm{~Hz})$ & 8.5 \\
\hline Number of Stereo-PIV snapshots & 2550 \\
\hline
\end{tabular}

Table 2: Main parameters of the Stereo-PIV database under consideration.

(2016)] (red line in the figure 11). We end up with a flow zone without bubbles which will be the Stereo PIV measurements zone illustrated in hatched yellow lines in the figure 11 . The bubbles are generated and then propagated out of this yellow zone (see the green circle in the figure 11). Our aim in the following is to study the hydrodynamic flow field in this yellow zone. This flow is responsible of the breaking wave generation that will be the origin of the bubble clouds generated behind the Stereo PIV measurements zone.

\section{Bubble clouds characterization}

In this section, we first describe the observed phenomenon through the experimental set-up presented above, from observations of the free surface behaviour at the bow, and of bubble clouds generation and propagation. Secondly, a 3D reconstruction of selected bubble clouds is proposed and their dynamical evolution is investigated. 


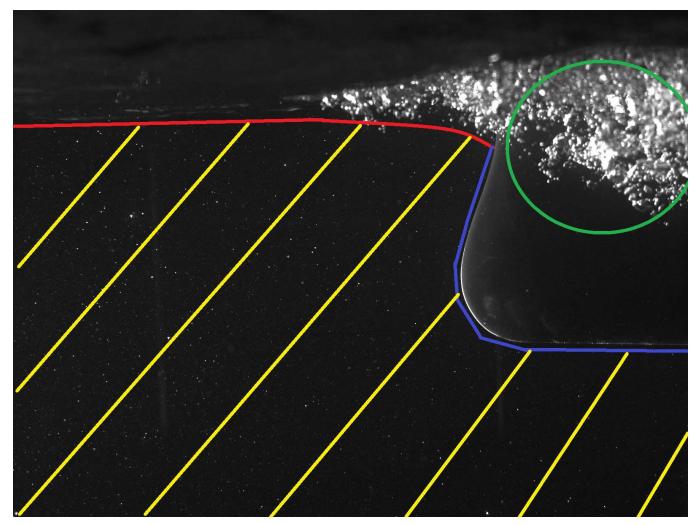

Figure 11: The Stereo PIV measurement zone.

\subsection{Description of the phenomenon}

For the 3D characterization of the phenomenon, measurements have been made over three minutes. The model has been submitted to the current $(\mathrm{U}=0.75 \mathrm{~m} / \mathrm{s})$, as well as synchronized waves $\left(33 \mathrm{~mm}\right.$ amplitude and $f_{0}=$ $0.85 \mathrm{~Hz}$ frequency) and ship motions ( $2 \mathrm{deg}$ pitch and $20 \mathrm{~mm}$ heave at $0.85 \mathrm{~Hz}$ ). Through this experimental configuration, air entrainment is generated due to breaking waves, coming from the interaction between the incoming waves and the reflected one on the bow and/or the impact between the bow and the free surface. A breaking wave is generated when the wave peak interacts with the flairing of the bow during plunging ship motions. The front of the bow wave advances considerably by forming a sheet at a distance of about $70 \mathrm{~mm}$ from the front of the ship bow (see figure 12a). After $0.23 \mathrm{~s}$, the bow wave comes closer to the hull and a second sheet appears. The distance between the two sheets is approximately $125 \mathrm{~mm}$ (see figure 12b). At this stage, the air entrainment takes place. The bubble cloud propagates along the hull and the bow wave is attenuated (see figure 12c) until it disappears. The duration of the phenomenon is about $0.47 \mathrm{~s}$ for a low intensity bubble cloud and about 0.82s for a high intensity bubble cloud.

The Figure 13 presents the image sequences taken by the three cameras during a wave period with bubbles around the classic bow of the $P p$ ?. Each raw image from 1 to 9 corresponds to the phase locations given in figure 13 (top-right). Images from cam 1 and cam2 show that the breaking wave takes place and begins at the front of the bow making an angle that reaches nearly $70 \mathrm{deg}$ in the phase 6 . The bubble cloud is generated (during phases 5 and 

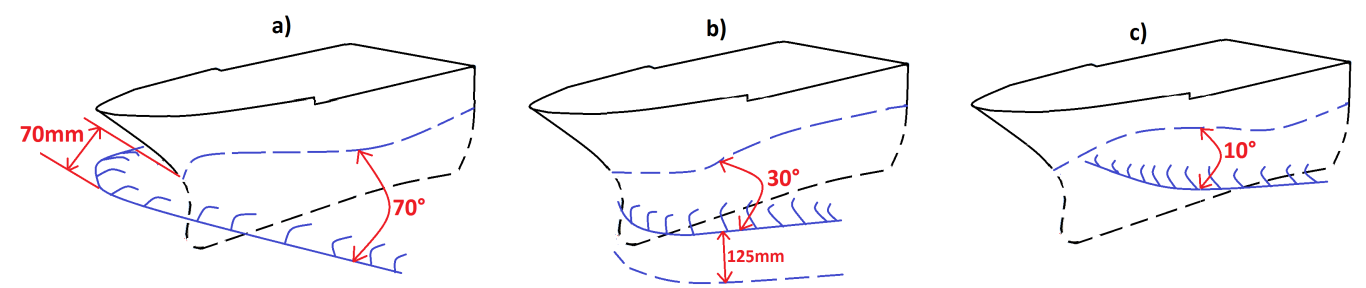

Figure 12: Description of the breaking wave mechanism.

6) and then propagated (during phases 7,8 and 9) along the ship hull as we can see in the images from cam3. The flairing of the bow of the $P p$ ? is the origin of an intense interaction between the incoming waves and the bow from which breaking waves are generated in front of the bow.

As detailed in the 3D reconstruction method in section II-2.1, we can reconstruct the bubble cloud in 3D from the combination between images from cam 1 and cam 3 as shown below. Images from cam3 enable to investigate the evolution of the bubble cloud in the $\mathrm{X}$ and $\mathrm{Z}$ directions and images from cam1 allow to identify the behaviour of the bubble cloud in the $\mathrm{Y}$ direction. 


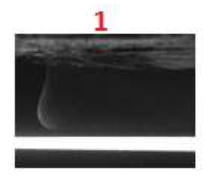

4

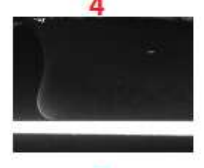

7

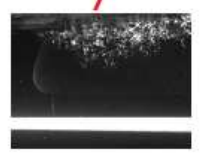

1

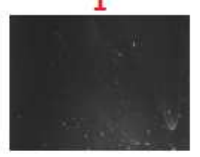

4

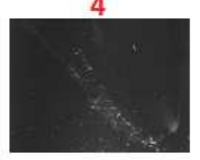

7

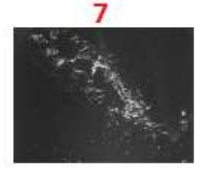

2

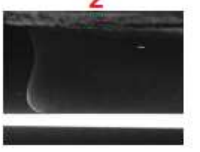

5

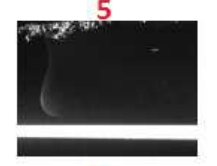

8

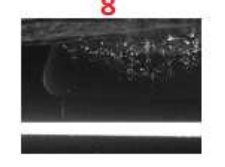

2

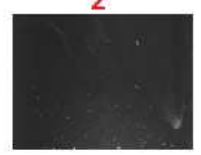

5

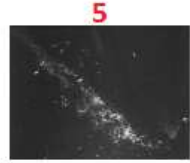

8

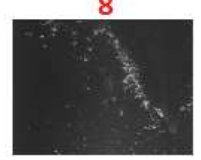

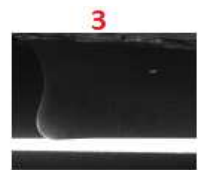

6

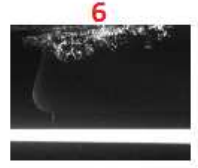

9

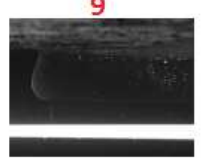

3

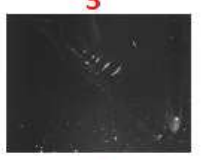

6

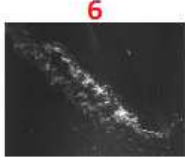

9

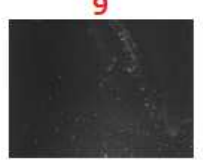

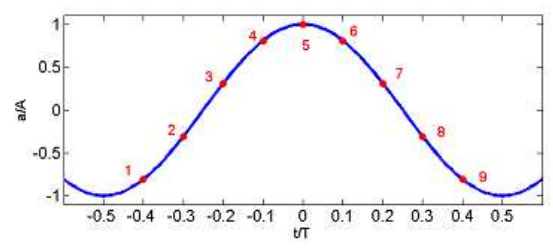

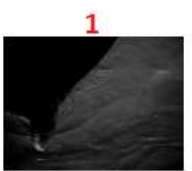

4

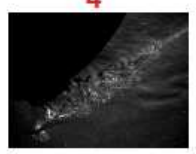

7

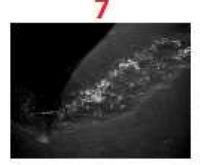

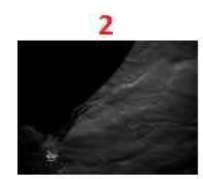

5

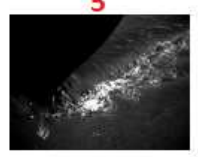

8

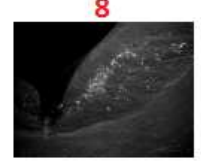

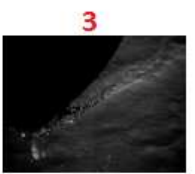

6

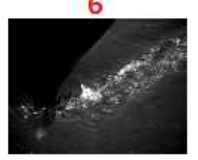

9

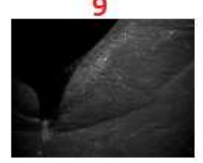

Figure 13: Images taken by cam 1 (bottom-left), cam2 (bottom-right) and cam3 (topleft). Each group of 9 graphs corresponds to nine consecutive instants of a same wave period with bubbles. The image 5 corresponds to the wave peak at the bow. Time evolution of the harmonic wave surface and the corresponding instants (top-right).

\subsection{Reconstruction and evolution of bubble clouds}

This experimental set-up allows us to visualize more precisely the bubble sweep-down phenomenon, comparing to previous studies [Delacroix et al. (Part 1 - 2016)]. The magnitude of the interaction between the free surface and the bow generates bow waves which are often the origine of bubble cloud generation. Therefore and as function of the breaking wave magnitude, several sizes of bubble clouds are observed. In order to characterize the 3D behaviour of bubble clouds generated by breaking waves, we choose only three of them encountered during one run. The first one is a low intensity bubble 
cloud, the second one is a medium bubble cloud and the third one is a high intensity bubble cloud. A low intensity bubble cloud is defined as a small cloud with a small volume which remains close to the free surface. A high intensity bubble cloud is defined as a big bubble cloud with a big volume which tends to go deeper. The occurrences for the different cloud sizes is almost the same. We detect about 102 occurrences for all sizes during the three minutes of recording, so it is about 34 occurrences/size. For figures $16,17,20,21,25$ and 26 below, each color corresponds to the position of the ship at a given instant. For instance, the blue bubble cloud is generated when the blue contour of the ship is in the blue position.

\subsubsection{Low intensity bubble cloud characterization}

For low intensity bubble cloud events, the totality of the cloud can be followed at each instant (see figure 14). We can see from the images of cam3, that the bubble cloud generated around the classic bow of the Pp? is not large in term of air quantity injected in the flow. Images from cam 1 show that the bubble cloud is generated (at $\mathrm{t} / \mathrm{T}=0.1$ and $\mathrm{t} / \mathrm{T}=0.2$ ) and propagated (at $\mathrm{t} / \mathrm{T}=0.3$ and $\mathrm{t} / \mathrm{T}=0.4$ ) close to the bow, where $\mathrm{T}$ is the wave period (see figure 13 showing the time evolution of the harmonic wave surface and the corresponding instants). The bubble cloud is reconstructed in 3D in the figure 15. The colors red, blue, green and black correspond respectively to the phases $6,7,8$ and 9 illustrated in the figure 13 . The bubble cloud reaches a maximum depth of $0.65 \mathrm{D}$ (see figure 16 left) and a maximum value of $0.37 \mathrm{~B}$ in the depth of field (see figure 16 right).

Figure 17 shows the evolution of the cloud propagation velocity components, calculated from the centroid of two succesive identified region positions. The centroid is determined from the bubble cloud center locations.

The three components $U_{\text {smallcloud }}, V_{\text {smallcloud }}$ and $W_{\text {smallcloud }}$ correspond to the bubble cloud propagation velocity in $\mathrm{X}, \mathrm{Y}$ and $\mathrm{Z}$ directions respectively. The instant $t=0$ in the figure 17 corresponds to the first cloud detection. The wave period is composed of two processes, the bubble cloud generation process (between phase 1 and 2 in figure 14) and the bubble cloud propagation process (between phase 2 and 4 in figure 14). The two processes are separated by a vertical dotted line in figure 17 . We can see that $V_{\text {smallcloud }}$ and $W_{\text {smallcloud }}$ components are always negative in this case. The bubble cloud horizontal velocity is almost constant $\left(U_{\text {smallcloud }}=0.73 \mathrm{~m} / \mathrm{s}\right)$ between phases 2 and 4 .

The uncertainty of the bubble cloud velocity is directly related to the 
uncertainty of the centroid position of the detected zone, and thus to the cloud area computation which directly related to the choice of the detection threshold. It has been shown that the choice of the threshold does not influence too much the results [Delacroix et al. (Part 2 - 2016)], one is left with an error of the order of $5 \mathrm{~cm}^{2}(6 \%)$ on the cloud area computation. Thus, the uncertainty of the bubble cloud velocity is about $6 \%$.
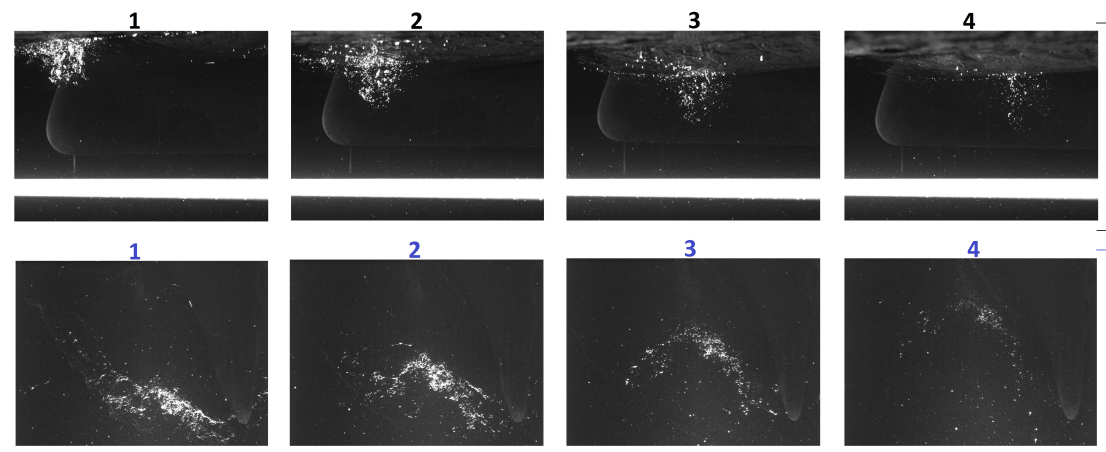

\section{cam3}
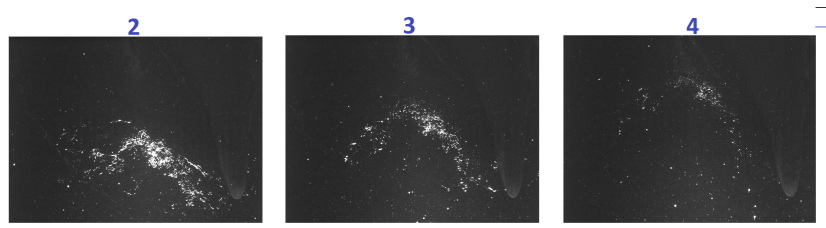

cam1

Figure 14: Images taken by cameras 1 and 3 during a wave period with a low intensity bubble cloud around the classic bow of $P p$ ?

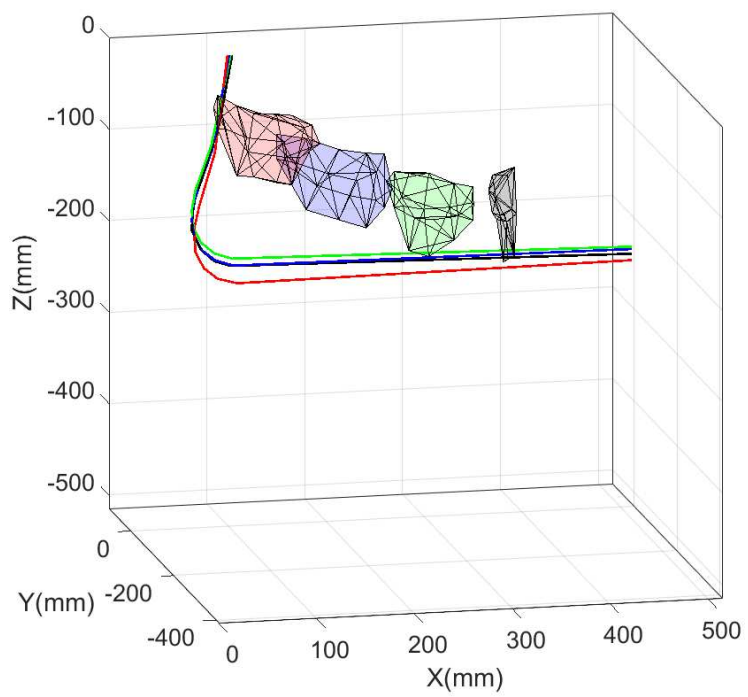

Figure 15: 3D reconstruction of the evolution of a low intensity bubble cloud around the classic bow of the $P p$ ? 

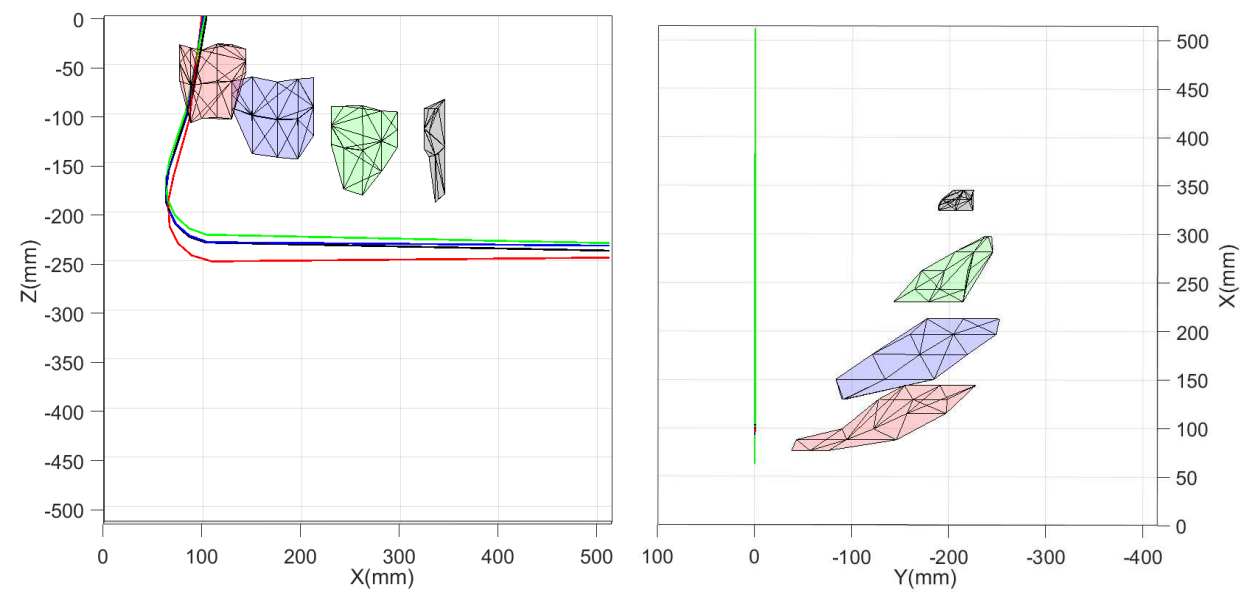

Figure 16: Reconstruction of the evolution of a low intensity bubble cloud around the classic bow of the $P p$ ? in $\mathrm{X}-\mathrm{Z}$ view (left) and in $\mathrm{Y}-\mathrm{X}$ view (right).

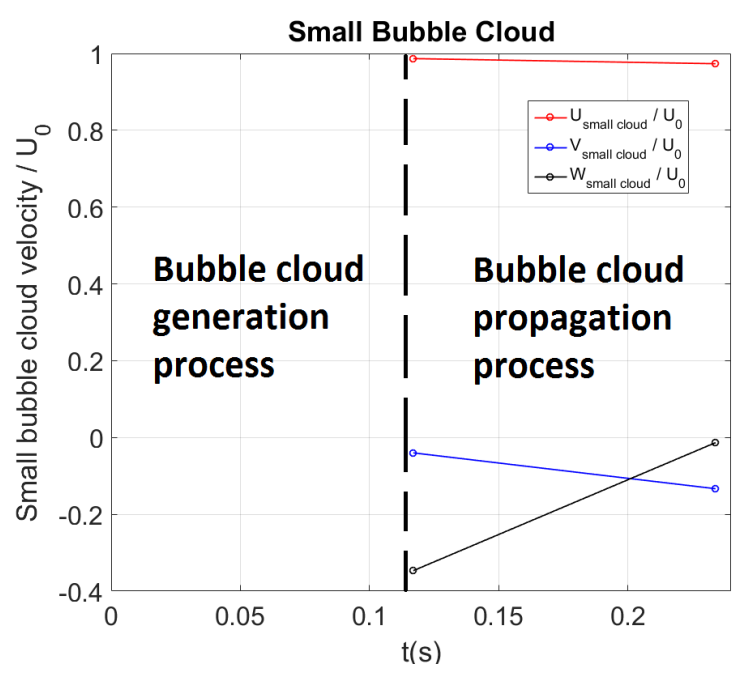

Figure 17: Low intensity bubble cloud velocity.

\subsubsection{Medium bubble cloud characterization}

A second kind of bubble cloud is presented here. Compared to the previous case, we can see from the images of cam3 that the medium bubble cloud generated around the classic bow of the $P p$ ? is more intense in terms of air quantity injected in the flow. Contrary to the previous case where the low intensity bubble cloud remains close to the bow, images from cam1 in 
the figure 18 show that the breaking wave takes place at the front of the bow, making an angle which reaches nearly $70 \mathrm{deg}$ in phase 6 , allowing to generate bubbles a little further from the bow. The 3D reconstruction presented in figure 19 shows a maximum depth of $0.83 \mathrm{D}$ (see figure 20 left). The bubble cloud reaches a maximum value of about $0.48 \mathrm{~B}$ in the transverse direction (see figure 20 right). In this case, the colors red, blue, green, black and pink correspond respectively to the phases 5, 6, 7, 8 and 9 illustrated in the figure 13 . The medium bubble cloud generation process also takes place between phases 1 and 2 which correspond to $t / T=0$ and $t / T=0.1$. It is then propagated during phase 2 to phase 5 (see figure 18) which correspond to $\mathrm{t} / \mathrm{T}=0.2, \mathrm{t} / \mathrm{T}=0.3$ and $\mathrm{t} / \mathrm{T}=0.4$. Figure 21 shows the evolution of the medium bubble cloud propagation velocity components.

We can see that $W_{\text {mediumcloud }}$ component are always negative in this case. The $V_{\text {mediumcloud }}$ component is positive (up to $0.25 U_{0}$ ) at the begining of the propagation process. The medium bubble cloud tends to go further in depth and in the transverse direction. The bubble cloud horizontal velocity oscillates between $U_{\text {smallcloud }}=0.6 \mathrm{~m} / \mathrm{s}$ and $U_{\text {smallcloud }}=0.73 \mathrm{~m} / \mathrm{s}$ between phases 2 and 5.
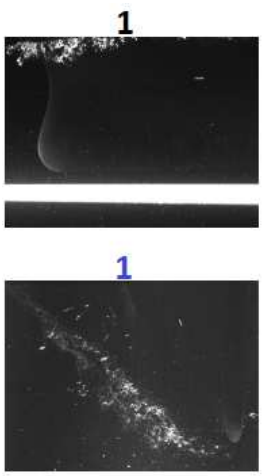

2

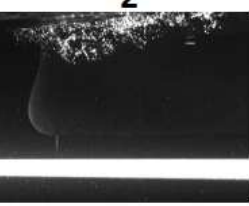

2

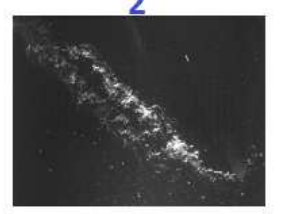

3

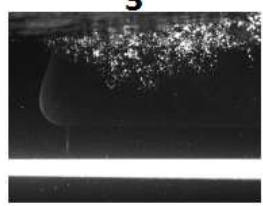

3

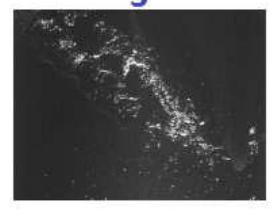

4

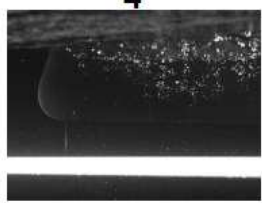

4

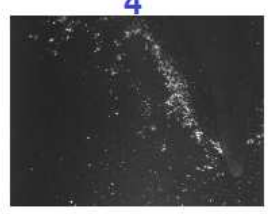

5

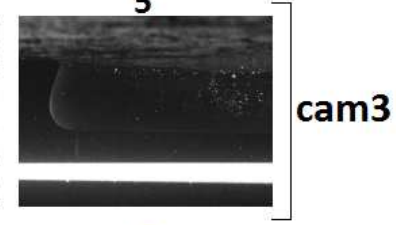

5

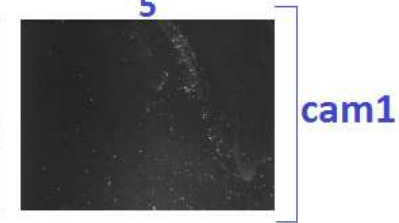

Figure 18: Images taken by cameras 1 and 3 during a wave period with a medium bubble cloud around the classic bow of $P p$ ? 


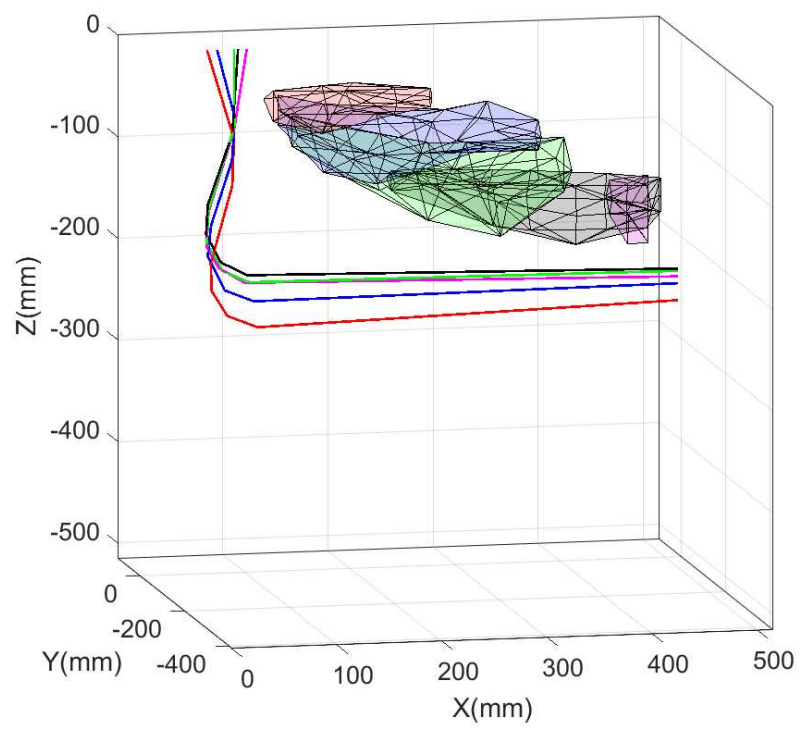

Figure 19: 3D reconstruction of the evolution of a medium bubble cloud around the classic bow of the $P p$ ?
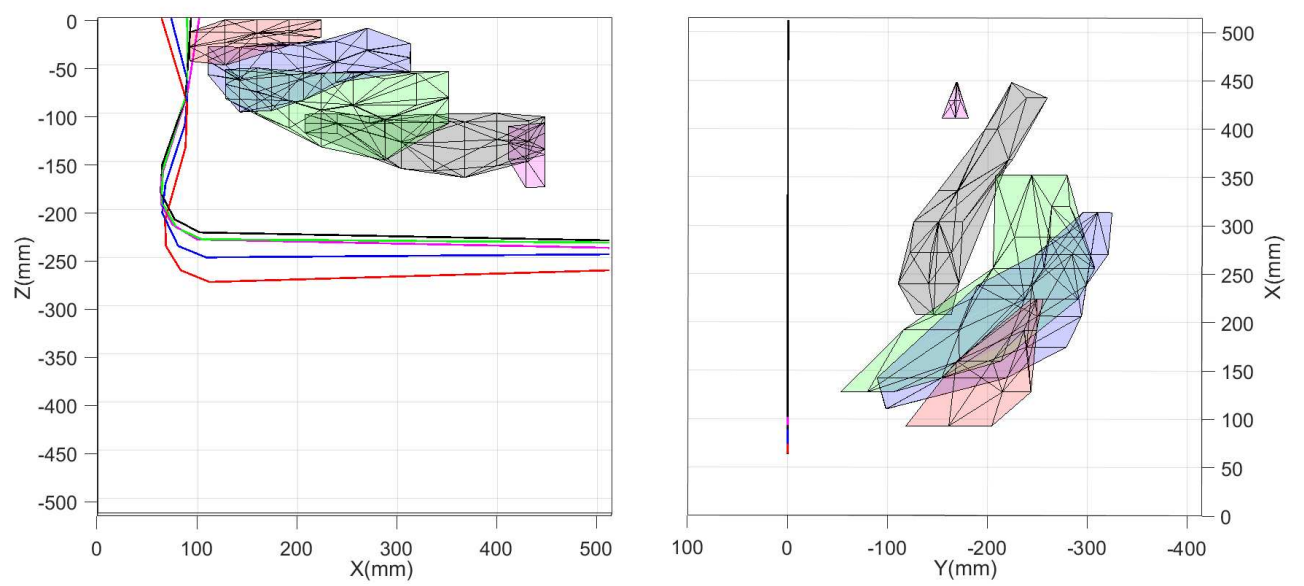

Figure 20: Reconstruction of the evolution of a medium bubble cloud around the classic bow of the $P p$ ? in $\mathrm{X}-\mathrm{Z}$ view (left) and in $\mathrm{Y}-\mathrm{X}$ view (right). 


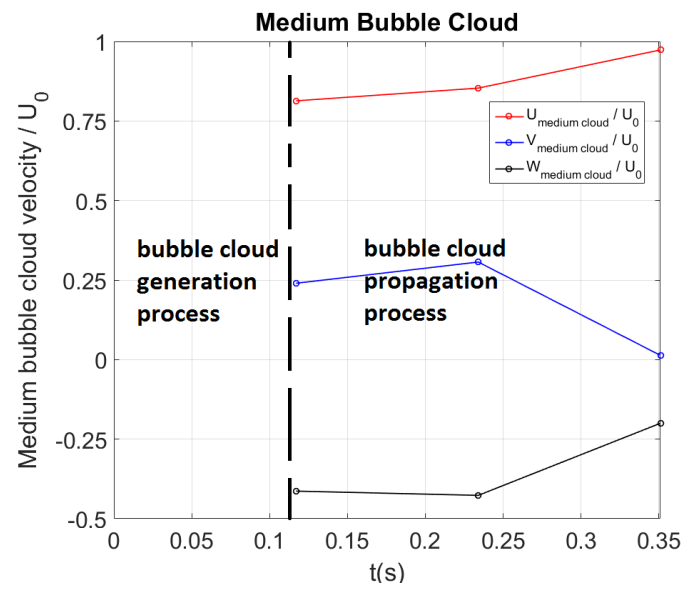

Figure 21: Medium bubble cloud velocity.

\subsubsection{High intensity bubble cloud characterization}

For the bigger events encountered during a run, it is hard to follow all the bubble cloud at each instant (see figure 22). Images from cam1 in the figure 22 shows that the breaking wave takes place in the front of the bow, making an angle which reaches nearly $90 \mathrm{deg}$ between phases 2 and 4 (see figure 23), allowing to generate bubbles far away from the bow. We can see from the images of cam3 that the air quantity injected in the flow is very consequent.

The 3D reconstruction presented in figure 24 shows a maximum depth of 1.1D (see figure 25 left). In this case, the colors red, blue, green, black and yellow correspond respectively to the phases 5, 6, 7, 8 and 9 illustrated in the figure 13. The high intensity bubble cloud reaches a maximum value of about $0.6 \mathrm{~B}$ in the transverse direction (see figure 25 right). The bubble cloud generation process takes place between phase 1 and phase 4 . It is then propagated during phase 4 to phase 7 (see figure 22). We note that the 3D reconstruction method is less adapted in this case: the size of the images is not adapted to these extreme events. Therefore, the bubble cloud center position is badly detected and the determination of the propagation velocity is not fully validated, reason why we don't present any cloud velocity results for this case. 

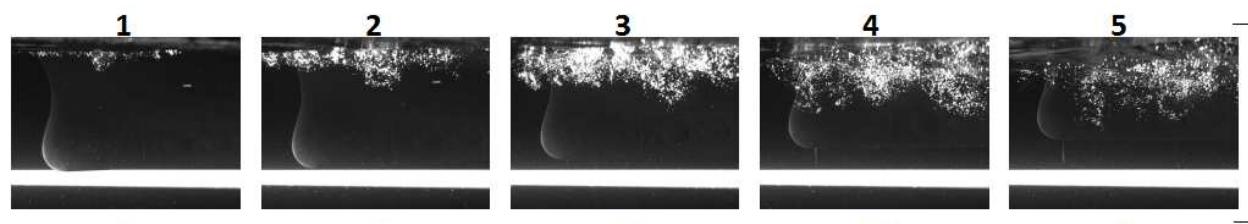

cam3
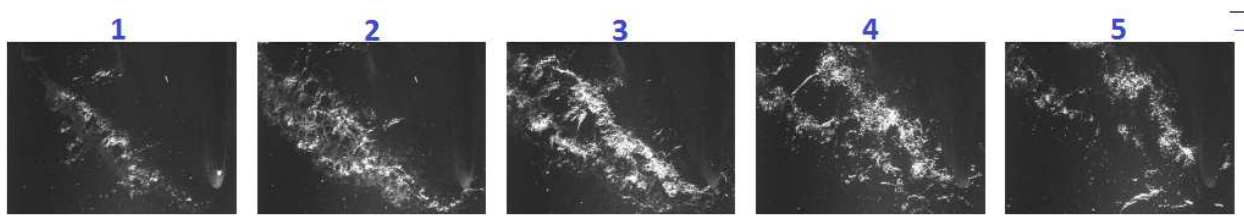

cam1
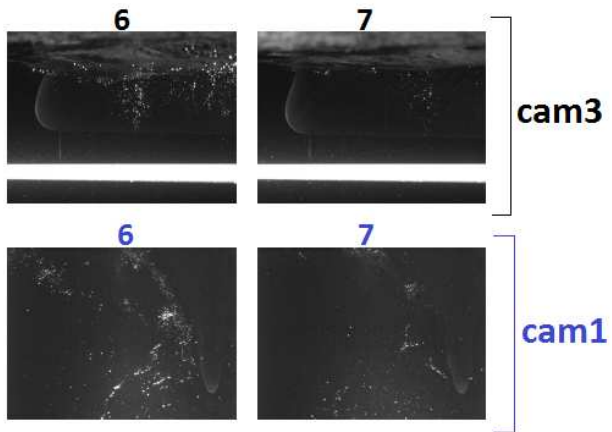

Figure 22: Images taken by cameras 1 and 3 during a wave period with a high intensity bubble cloud around the classic bow of $P p$ ?

1

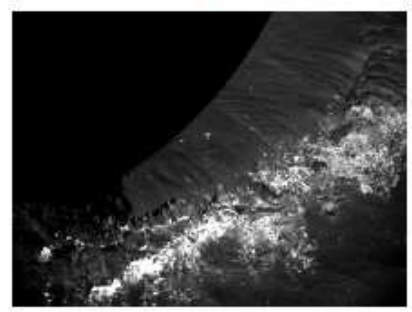

2

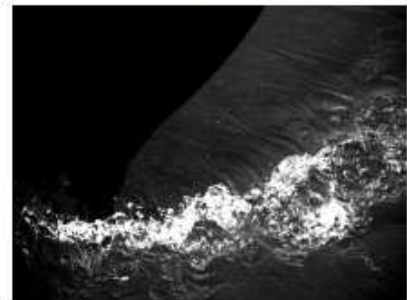

3

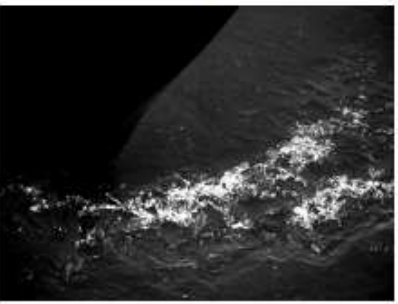

Figure 23: Images taken by camera 2 during a wave period with a high intensity bubble cloud around the classic bow of $P p$ ? 


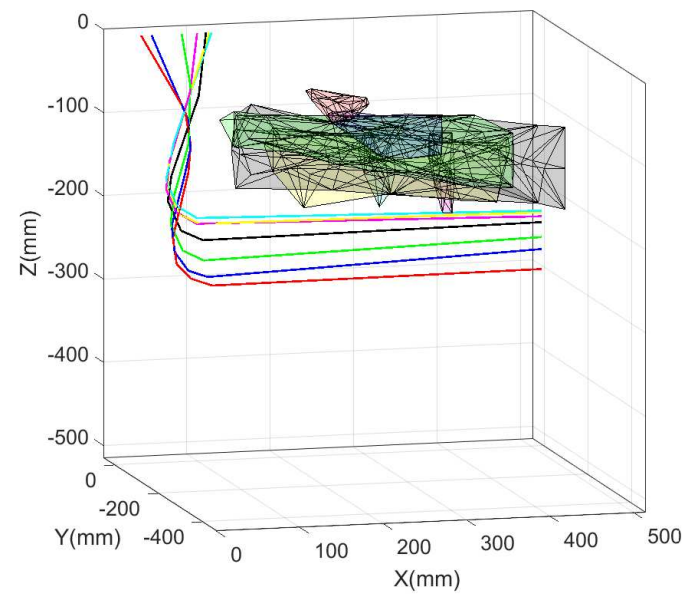

Figure 24: 3D reconstruction of the evolution of a high intensity bubble cloud around the classic bow of the $P p$ ?
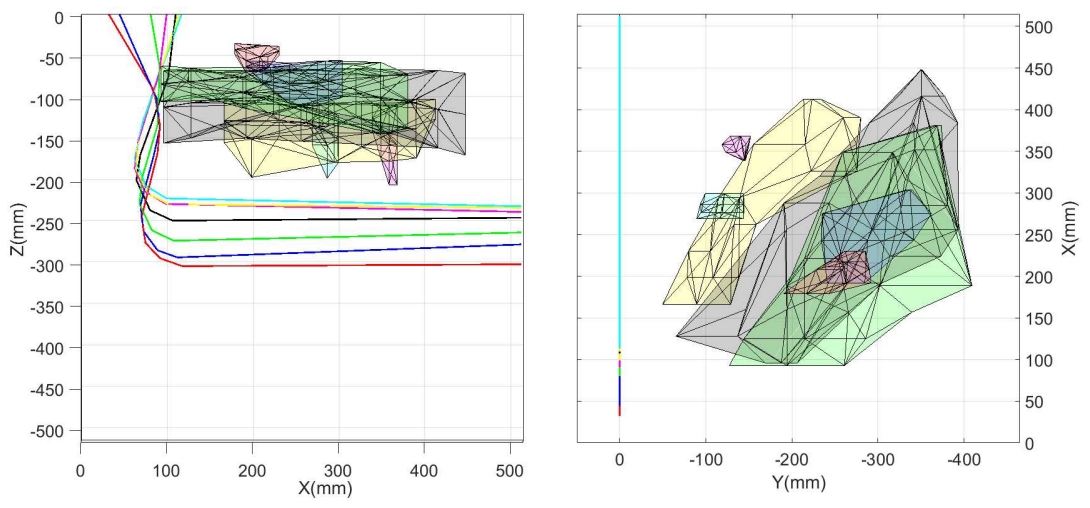

Figure 25: Reconstruction of the evolution of a high intensity bubble cloud around the classic bow of the $P p$ ? in $\mathrm{X}-\mathrm{Z}$ view (left) and in $\mathrm{Y}-\mathrm{X}$ view (right).

From all these observations, we note that the 3D reconstruction method of the bubble cloud and its evolution along the ship hull allow a good characterization of the bubble sweep-down phenomenon. We are now able to follow the evolution of the bubble cloud in the transverse direction thanks to the camera fixed at the bottom of the flume tank. We note that we did not have access to this information in previous 2D studies [[Delacroix et al. (Part 2 2016)]]. The characterization of the bubble cloud in the horizontal plane is very usefull and allows to determine if bubble clouds are propagated under 
the ship hull.

Three types of bubble clouds have been characterized from generation to propagation processes. The reconstruction method is well implemented for low intensity and medium bubble clouds. We can follow all the bubble cloud once generated. For the high intensity events, the method is less adapted because the clouds occupy more than the whole image and it is hard to follow the cloud at each instant. The bubble cloud generation process takes more time for the high intensity bubble cloud compared to the low intensity and medium bubble cloud generation process.

The bubble cloud propagation velocity shows that the low and medium intensity bubble clouds have almost the same behaviour in $\mathrm{X}$ and $\mathrm{Z}$ directions. A value of around $0.8 U_{0}$ for the longitudinal velocity component and a value of about $-0.4 U_{0}$ for the vertical velocity component are noted. Regarding the transverse velocity component $\mathrm{V}$, the propagation of the bubble cloud in the $\mathrm{Y}$ direction is faster for the medium bubble cloud compared to the low intensity bubble cloud.

Finally, we note that for the cases of the medium and high intensity bubble clouds where intense breaking waves are generated, the clouds tend to go further not only in depth, but also transversely allowing a propagation along the hull and near the transducer. Acoustic surveys can be disrupted in these cases due to the high occurrence frequency and dense bubble clouds especially in this configuration with current, waves and ship motions [Mallat et al. (2017)].

\section{Flow analysis}

In this section, we present the Stereo-PIV analyses performed in the ship axis of symmetry where breaking waves take place. Indeed, StereoPIV investigation in such a flow configuration with waves and ship motions can be problematic because of the presence of the oscillating wave surface, of bubbles and of many reflections. In order to overcome these effects, we first detect automatically the unsteady free water-air interface [Dussol et al. (2016)]. Due to the difficulty to analyse the instantaneous velocity fields, a POD analysis is performed to reconstruct the phase average velocity field from each available PIV velocity field in order to identify the flow motion that is significantly responsible for the air entrainment. The POD analysis proposed here is only a denoising tool that allows to smooth the velocity fields. 
POD is performed from the $N t=2550$ instantaneous longitudinal (Upod), transverse (Vpod) and vertical (Wpod) components of the velocity field accessed on a regular grid mesh of $n x \times n z=93 \times 69$ points. The energetic content of the first three POD modes represents about $82 \%$ of the total energy. The first POD spatial eigenfunctions as well as the first POD temporal coefficients are analyzed similarly to previous works ([Druault et al. (2014)]). Then it is demonstrated that the projection of each instantaneous velocity field onto the first three POD modes gives an instantaneous dominant flow field that corresponds to the phase average velocity field.

Figures 26 and 27 show the three components of the velocity vector field without and with bubble generation respectively. A wave period with bubbles is defined as a period where the wave is broken and then the bubble clouds generation is observed. The wave periods without bubbles are the periods where there is no bubble generation. The three components Upod, Vpod and Wpod correspond to the flow velocity in the $\mathrm{X}, \mathrm{Y}$ and $\mathrm{Z}$ directions respectively. Each image from 1 to 9 corresponds to the average of the velocity fields in all periods without or with bubbles and corresponds to the phases provided in figure 13 .
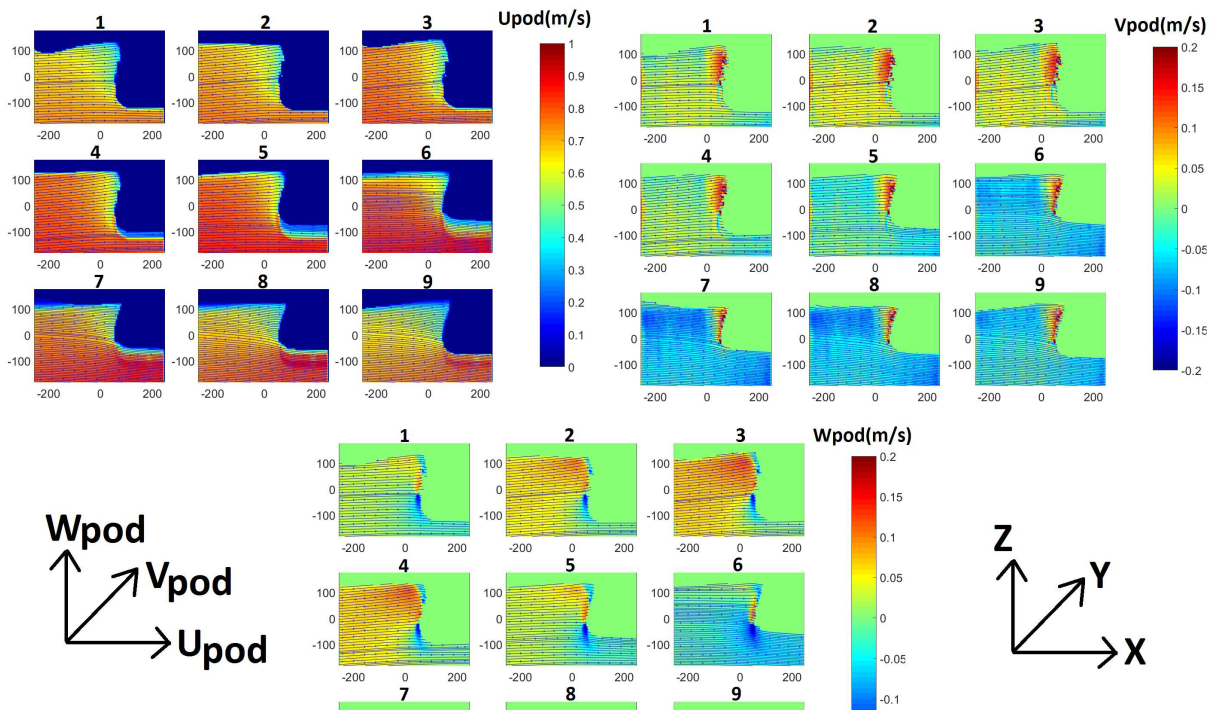

$\operatorname{Wpod}(\mathrm{m} / \mathrm{s})$
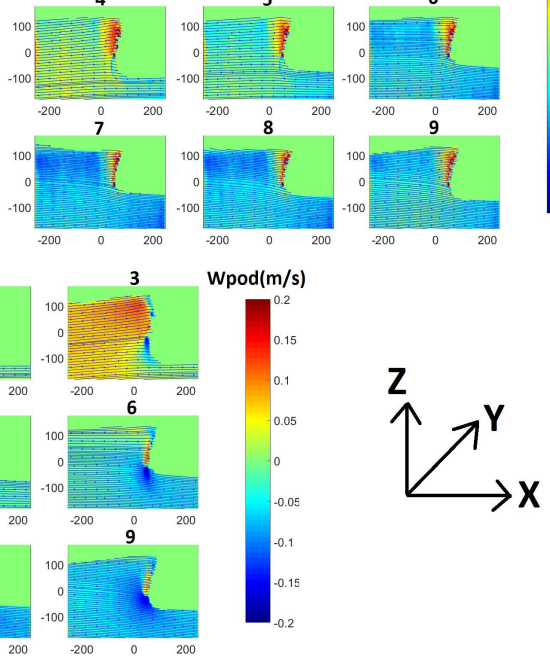

Figure 26: Representation of the nine consecutive velocity fields (without bubbles) deduced from the projection of velocity field onto the first three POD modes. 

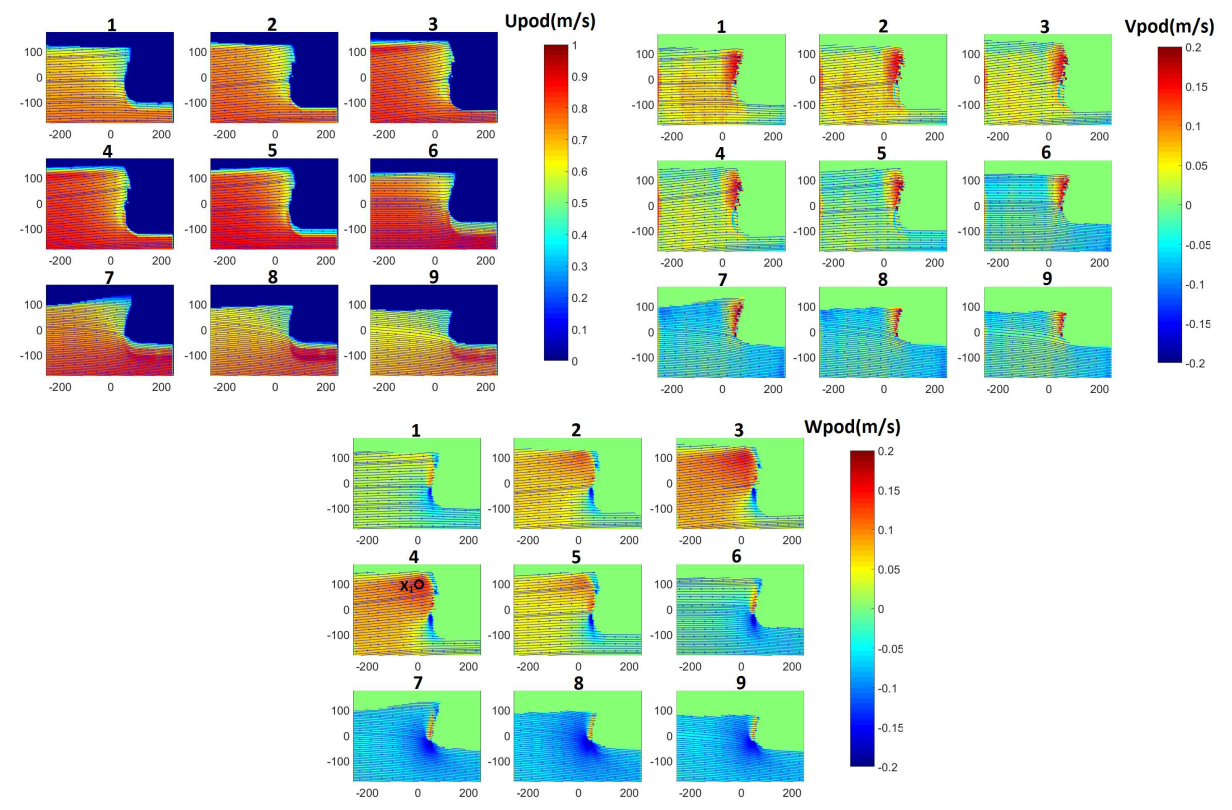

Figure 27: Representation of the nine consecutive velocity fields (with bubbles) deduced from the projection of velocity field onto the first three POD modes.

We note that the positive vertical velocities (Wpod) during surface elevation are higher in the contact zone between the shape and the surface for the case with bubbles, with a maximal measured velocity of about $0.2 \mathrm{~m} / \mathrm{s}$ above the bulb of the hull in the phase 4 . The transverse velocity component (Vpod) is almost zero. Finally for the longitudinal velocity component (Upod), we obtain a value between 0.5 and $0.8 \mathrm{~m} / \mathrm{s}$ for the two cases.

To confirm these observations, figure 28 presents the temporal evolution of the three velocity components $u_{1}, v_{1}$ and $w_{1}$ at the point $X_{1}$ located above the bulb of the bow as shown in figure 27 (phase 4 for the w component). The circles in figure 28 correspond to the bubbly cases. For these cases, we note that the variabilty of the velocity fields can be consequent. For instance, a bubble cloud can be generated for a horizontal velocity of $0.5 \mathrm{~m} / \mathrm{s}$, as for a horizontal velocity of $0.8 \mathrm{~m} / \mathrm{s}$. Figure 29 shows the evolution of the three velocity components of the phase 4 at the point $X_{1}$ for these bubbly cases (on the left) and for the non-bubbly cases (on the right). The three velocity components are more consequent in the case with bubbles especially for the vertical velocity component $w_{1}$. For instance, we obtain the mean values of $0.15 \mathrm{~m} / \mathrm{s}$ and $0.09 \mathrm{~m} / \mathrm{s}$ for the $w_{1}$ component with and without bubbles 
respectively. Regarding the transverse velocity component $v_{1}$, there is no significant difference between the two cases, with a mean value close to zero. The mean velocity components $\overline{u_{1}}, \overline{v_{1}}$ and $\overline{w_{1}}$ extracted at the point $X_{1}$ with and without bubbles in the phase 4 are summarized in table 3 .

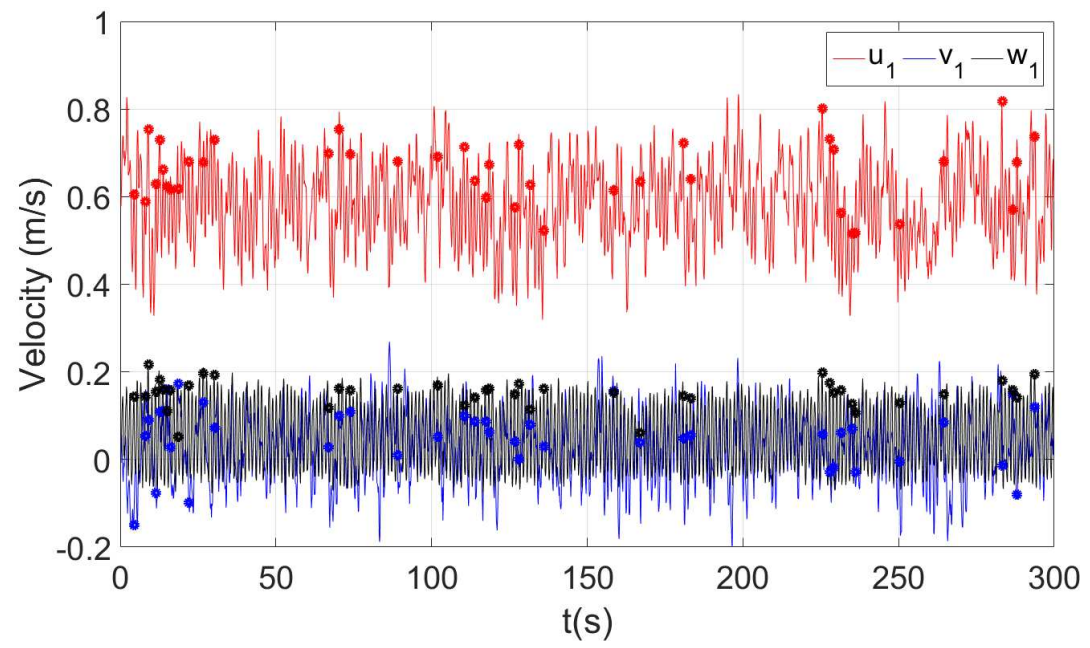

Figure 28: The evolution of the three velocity components at the local point $X_{1}$.
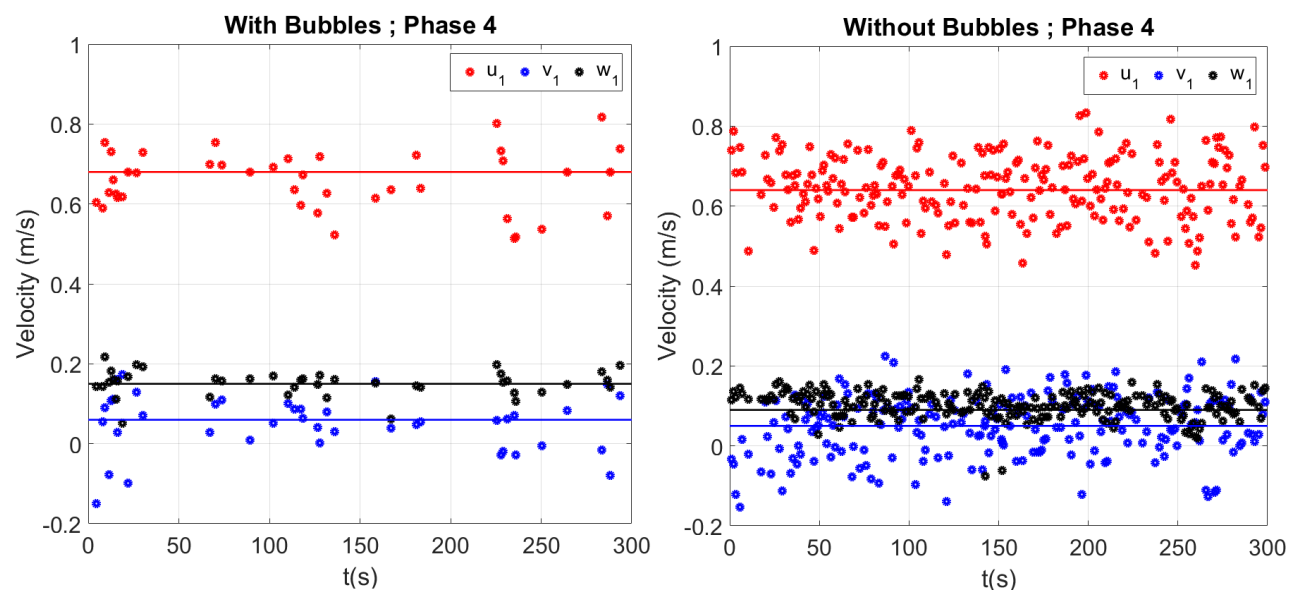

Figure 29: The evolution of the three velocity components in the phase 4 at the local point $X_{1}$. Left: bubbly cases. Right: non-bubbly cases. The horizontal lines correspond to the mean value. 


\begin{tabular}{|c|c|c|c|}
\hline & $\overline{u_{1}}(\mathrm{~m} / \mathrm{s})$ & $\overline{v_{1}}(\mathrm{~m} / \mathrm{s})$ & $\overline{w_{1}}(\mathrm{~m} / \mathrm{s})$ \\
\hline With bubbles & 0.68 & 0.06 & 0.15 \\
\hline Without bubbles & 0.64 & 0.05 & 0.09 \\
\hline
\end{tabular}

Table 3: The different values of the mean average velocity components at the local point $X_{1}$ in the phase 4 for the bubbly and non-bubbly events.

These observations reveal that the bubble generation is mainly conditioned by the ascending vertical velocity $\left(w_{1}\right)$ of the flow (phase 4 ) concentrated above the bulb of the bow. The high ascending velocities are the origin of intense breaking waves, causing the generation of bubbles between phases 5 and 7 which are pulled and propagated by the flow during phases 7 and 9 .

The results presented in this section allow to characterize the flow around the bow and are complementary to the 3D visualization of the bubble sweepdown phenomenon in section III. These measurements are representative of the flow at real scale thanks to the Froude similitude while some differences can occur on air bubble detection due to Webber effects.

\section{Conclusion}

An experimental study of the bubble sweep-down phenomenon around a 1/30 scale ship model has been carried out in a wave and current circulating tank allowing a 3D characterization of the phenomenon. Three synchronized cameras located around the ship bow with an horizontal laser sheet enabled to visualize the bubble sweep-down phenomenon from different points of view. A 3D reconstruction method has been proposed to follow the evolution of the bubble clouds, generated and propagated around the ship model.

This experimental study allows a global visualization and characterization of the bubble sweep-down phenomenon compared to previous 2D studies (Mallat et al. (2017)) for which we do not have access to the transverse behaviour of the bubble cloud. The images from the camera at the bottom of the tank show that breaking waves take place in the front of the bow making a large angle with the bow that reaches nearly 70 deg during a wave period with bubbles.

Three types of bubble clouds have been investigated by applying the $3 \mathrm{D}$ reconstruction method. The results show that for the bow intensity and medium bubble clouds, the entire cloud is identified from generation to propagation processes. The horizontal and vertical propagation velocities of these 
bubble clouds are almost the same. However, the meddium bubble cloud is propagated faster in the transverse direction. Regarding the high intensity bubble cloud, the breaking wave is large and the reconstruction method is less adapted. We observe that the bubble cloud tends to go further not only in depth, but also in transverse direction allowing a propagation all around the hull. The bubble clouds generation process is longer for the high intensity bubble cloud compared to low intensity and medium bubble clouds.

To complete the 3D visualization of the phenomenon, Stereo-PIV measurements followed by POD analyses have been performed in the main axis of symmetry of the model. The wave periods with and without bubbles have been analysed. The results reveal that the bubble generation is mainly conditioned by the ascending vertical velocity of the flow prior to the formation of bubble clouds. These ascending velocities are the origin of the breaking wave, causing the formation of bubbles which are entrained by the flow. There is no notable difference of the longitudinal and transverse velocity components between the cases with and without bubbles.

The bubble sweep-down phenomenon is complex for which bubbles are fairly scattered in the three dimensions of space once the wave is broken. Even though the velocity maps are representative at real scale through the Froude similitude, a complementary trials should be conducted by using an optical probe to measure the void fraction, the bubbles size and velocity around the ship hull. Despite the complexity of the phenomenon, we are now able to optimize the position of the optical probe around the ship model thanks to the 3D characterization done in this paper.

\section{Acknowledgements}

The authors would like to thank Ifremer and the Hauts-de-France Regional Council for the financial funding of the PhD project. We are also grateful to Thomas Bacchetti and Jean-Valery Facq for their assistance in the design and set-up of these experiments.

\section{References}

Dalen, J., Lovik, A. (1981). The influence of wind-induced bubbles on echo integration surveys, J. Acoust.Soc. Am., Vol.69, 1653-1659. 
Delacroix S., Germain G., Berger L., Billard, J-Y. (2016). Bubble sweepdown occurrence characterization on Research Vessels. Ocean Engineering $111,34-42$.

Shabangu, F. W., Ona, E., Yemane, D. (2014). Measurements of acoustic attenuation at $38 \mathrm{kHz}$ by wind induced air bubbles with suggested correction factors for hull-mounted transducers, Fisheries research, Vol.151, 47-56.

Moraga, F.J., Carrica, P.M., D.A., Drew, R.T., Lahey, Jr. (2008). A sub-grid air entrainment model for breaking bow waves and naval surface ships. Computer \& Fluids, 37:281-298.

Johansen, J.P. (2010). Full-scale two-phase flow measurements on Athena research vessel, $\mathrm{PhD}$ Thesis of university of IOWA.

Castro A.M., Li J., Carrica P.M. (2016). A mechanistic model of bubble entrainment in turbulent free surface flow. Int. Journal of Multiphase Flow.

Noblesse F., Delhommeau G., Guilbaud M., Hendrix D., Yang C. (2008). Simple analytical relations for ship bow waves, Journal of Fluid Mechanics, Vol.600, 105-132.

Noblesse F., Delhommeau G., Guilbaud M., Liu H., Wan D., Yang C. (2013). Ship bow waves, Journal of Hydrodynamics, Vol.25, 491-501.

Delhommeau G., Guilbaud M., David L., Yang C., Noblesse F. (2009). Boundary between unsteady and overturning ship bow wave regimes, Journal of Fluid Mechanics, Vol.620, 167-175.

Kim, W. J., Van, S. H., Kim, D. H. (2001). Measurements of flows around modern commercial ship models. Experiments in Fluids, 31:567-578.

Chen, J. H., Chang, C. C. (2006). A moving PIV system for ship model test in a towing tank. Ocean Engineering, 33(14-15):2025-2046.

Lee, S. J., Koh, M. S., Lee, C. M. (2003). PIV velocity field measurements of flow around a kriso 3600teu container ship model. Journal of Marine Science and Technology, 8(2):76-87.

Lee, J. Y., Paik, B. G., Lee, S. J. (2009). PIV measurements of hull wake behind a container ship model with varying loading condition. Ocean Engineering, 36(5):377-385. 
Paik, B. G., Kim, J., Park, Y. H., Kim, K. S., Yu, K. K. (2007). Analysis of wake behind a rotating propeller using PIV technique in a cavitation tunnel. Ocean Engineering, 34(3-4):594-604.

Gomit, G., Chatellier, L., Calluaud, D., David, L. (2013). Free surface measurement by stereo-refraction. Experiments in Fluids, 54(6):1-11.

Gomit, G., Chatellier, L., Calluaud, D., David, L., Fréchou, D., Boucheron, R., Perelman, O., Hubert, C. (2015). Large-scale free surface measurement for the analysis of ship waves in a towing tank. Experiments in Fluids, $56: 1-13$.

Delacroix S., Germain G., Gaurier B., Billard J-Y. (2016). Experimental study of bubble sweep-down in wave and circulating tank. Part I - Experimental set-up and observed phenomena. Ocean Engineering 120, 78-87.

Delacroix S., Germain G., Gaurier B., Billard J-Y. (2016). Experimental study of bubble sweep-down in wave and circulating tank. Part II - Bubble clouds characterization. Ocean Engineering 120, 88-99.

Mallat, B., Germain, G., Gaurier, B., Druault, P., Billard, J-Y. (2018). Experimental Study of the Bubble Sweep-down Phenomenon on Three Bow Designs. Ocean Engineering 148, 361-375.

Raffel, Markus, et al. Particle Image Velocimetry: a practical guide. Springer, 2013.

Druault, P., Bouhoubeiny, E., Germain, G. (2014). Phase-averaged mean properties of turbulent flow developing around fluttering sheet of net. Ocean Engineering 82, 160-168.

Dussol, D., Druault, P., Mallat, B., Delacroix, S., Germain, G. (2016). Automatic dynamic mask extraction for PIV images containing an unsteady interface, bubbles, and a moving structure. C.R. Mecanique 344, 464-478. 